\title{
Determining the optimal area-dependent blank holder forces in deep drawing using the response surface method
}

\author{
Volk, M. ${ }^{a,}{ }^{,}$, Nardin, B. ${ }^{a}$, Dolsak, B. ${ }^{b}$ \\ ${ }^{a}$ Gorenje Orodjarna, d.o.o., Velenje, Slovenia \\ ${ }^{b}$ University of Maribor, Faculty of Mechanical Engineering, Maribor, Slovenia
}

\begin{abstract}
A B S T R A C T
Metal forming processes are often currently highly automated mass production processes for manufacturing a wide variety of metal parts from various industries. Maximizing product quality and consequently minimizing waste and production costs are major goals for those companies exploiting metal forming processes. On the other hand, sheet metal parts become more complex especially because of complex product designs and the usages of higher strength steels that have less formability. Therefore, metal forming processes need to be optimized. This research study demonstrates an optimization system for optimizing the sheet metal forming process using the Finite Element Method (FEM) combined with the Response Surface Method (RSM). The proposed optimization system was tested on an industrial example from the household appliances industry. In this study, it is described as to how to determine optimal area-dependent blank-holder forces in deep drawing process in order to obtain the best possible quality of the drawing part. The optimization system consists of three main steps: modeling, screening, and optimization. The results showed that with better preferences regarding the blankholder forces, better results can be achieved. Forming and spring-back criteria were taken into account. The number of required numerical simulations using the RSM combined with the Design of Experiment was not critical and was much smaller than using other conventional optimization methods. Therefore, reasonably accurate results can be achieved in a relativity short time, which is one of the main advantages of this method.
\end{abstract}

\section{ARTICLE INFO}

Keywords:

Sheet metal forming

Optimization

Finite element method

Response surface method

*Corresponding author: mihael.volk@gorenje-orodjarna.si (Volk, M.)

Article history:

Received 6 December 2013

Revised 30 May 2014

Accepted 3 June 2014 


\section{References}

[1] Tisza, M. (2013). Recent development trends in sheet metal forming, International Journal of Microstructure and Materials Properties, Vol. 8, No. 1/2, doi: 10.1504/IJMMP.2013.052651.

[2] Albut, A., Ciubotaru, V., Radu, C., Olaru, I. (2011). Optimization of the blank holder force using neural network algorithm, In: AIP Conf. Proc. 1383, Vol. 1383, 1004-1009, doi: 10.1063/1.3623714.

[3] Liewald, M., Wurster, K., Blaich, C. (2011). New approaches on automated wrinkle detection in sheet metal components by forming simulation, In: AIP Conf. Proc. 1353, 1185-1190, doi: 10.1063/1.3589677.

[4] Gantar, G., Kuzman, K., Filipič, B. (2005). Increasing the stability of the deep drawing process by simulationbased optimization, Journal of Materials Processing Technology, Vol. 164-165, 1343-1350, doi: 10.1016/i. imatprotec.2005.02.099.

[5] Kitayama, S., Hamano, S., Yamazaki, K., Kubo, T., Nishikawa, H., Kinoshita, H. (2010). A closed-loop type algorithm for determination of variable blank holder force trajectory and its application to square cup deep drawing, The International Journal of Advanced Manufacturing Technology, Vol. 51, No. 5-8, 507-517, doi: 10.1007/s00170010-2656-9.

[6] Palaniswamy, H., Braedel, M., Thandapani, A., Altan, T. (2006). Optimal programming of multi-point cushion systems for sheet metal forming, CIRP Annals - Manufacturing Technology, Vol. 55, No. 1, 249-254, doi: 10.1016/S0007-8506(07)60409-0.

[7] Neugebauer, R., Leib, U., Bräunlich, H., (1997). Influence on materials flow in deep drawing using individual controllable draw pins and smooth blankholder design, In: Proceedings of SAE International Congress \& Exposition, Detroit, Michigan, SEA Paper No. 970989, 269-274, doi: 10.4271/970989.

[8] Endelt, B., Tommerup, S., Danckert, J. (2009). A novel feedback control system - Controlling the material flow in deep drawing using distributed blank-holder force, Journal of Materials Processing Technology, Vol. 213, No. 1, 36-50, doi: 10.1016/i.jmatprotec.2012.08.003.

[9] Volk, M., Deželak, M., Nardin, B., Stepišnik, S. (2012). Prediction of the spring-back calculated with numerical simulations for the household industry, In: AIP Conf. Proc. 1383, 1078-1085, doi: 10.1063/1.3623723.

[10] Deželak, M., Pahole, I., Stepišnik, A., Fijavž, R. (2011). Finite element method combined with machine learning for springback prediction, In: $6^{\text {th }}$ International Conference and Exhibition on Design and Production of Machines and Dies/Molds, Atilim University, Ankara, Turkey, 83-87.

[11] Dezelak, M., Stepisnik, A., Pahole, I., Ficko, M. (2014). Evaluation of twist springback prediction after an AHSS forming process, International Journal of Simulation Modelling, Vol. 13, No. 2, 171-182, doi: 10.2507/ IJSIMM13(2)4.261.

[12] Tang, L., Wang, H., Li, G. (2013). Advanced high strength steel springback optimization by projection-based heuristic global search algorithm, Materials \& Design, Vol. 43, 426-437, doi: 10.1016/i.matdes.2012.06.045.

[13] Volk, M., Nardin, B., Dolšak, B. (2011). Application of numerical simulations in the deep-drawing process and the holding system with segments' inserts, Strojniški vestnik - Journal of Mechanical Engineering, Vol. 57, 697-703, doi: $10.5545 /$ sv-jme.2010.258.

[14] Albut, A., Ciubotaru, V., Radu, C., Olaru, I. (2011). Optimization of the blank holder force using the neural network algorithm, In: AIP Conf. Proc. 1383, 1004-1009, doi: 10.1063/1.3623714.

[15] Intarakumthornchai, T., Jirathearanat, S., Juntaratin, J. (2011). Determination of loading paths in hydromechanical deep drawing process of parabolic cup with FEA based 2-D interval halving and fuzzy logic, In: Proceedings of the $4^{\text {th }}$ International Conference on Modelling and Simulation of Metallurgical Processes in Steelmaking - Metec Insteelcon 2011, Düseldorf, Germany.

[16] Kitayama, S., Kita, K., Yamazaki, K. (2012). Optimization of variable blank holder force trajectory by sequential approximate optimization with RBF network, The International Journal of Advanced Manufacturing Technology, Vol. 61, No. 9-12, 1067-1083, doi: 10.1007/s00170-011-3755-y.

[17] Montgomery, D.C. (2012). Design and analysis of experiments, $5^{\text {th }}$ ed., Wiley, New York.

[18] Bonte, M.H.A. (2007). Optimisation strategies for metal forming processes, PhD Thesis, University of Twente, Enschede, The Netherlands.

[19] Intarakumthornchai, T., Jirathearanat, S., Thongprasert, S., Dechaumphai, P. (2010). FEA based optimization of blank holder force and pressure for hydromechanical deep drawing of parabolic cup using 2-D interval halving and RSM methods, Engineering Journal, Vol. 14, No. 2, 15-32, doi: 10.4186/ej.2010.14.2.15.

[20] Velmanirajan, K., Thaheer, A.S.A., Narayanasamy, R., Ahamed Basha, C. (2012). Numerical modelling of aluminium sheets formability using response surface methodology, Materials \& Design, Vol. 41, 239-254, doi: 10.1016/ j.matdes.2012.05.027.

[21] Sun, G., Li, G., Gong, Z., Cui, X., Yang, X., Li, Q. (2010). Multiobjective robust optimization method for drawbead design in sheet metal forming, Materials \& Design, Vol. 31, No. 4, 1917-1929, doi: 10.1016/j.matdes.2009.10.050.

[22] Volk, M. (2013). Stabilization of the deep drawing process with sheet metal holding control, PhD Thesis, (original title: Stabilizacija procesa globokega vleka z obvladovanjem pridrževanja pločevine), University of Maribor, Faculty of mechanical engineering, Maribor, Slovenia.

[23] Hribernik, A. (2011). Methods of experimental work: design of experiments (original title: Metode eksperimentalnega dela: načrtovanje eksperimentov), University of Maribor, Faculty of mechanical engineering, Maribor, Slovenia.

[24] Abspoel, M., Scholting, M.E., Droog, J.M.M. (2012). A new method for predicting forming limit curves from mechanical properties, Journal of Materials Processing Technology, Vol. 213, No. 5, 759-769, doi: 10.1016/ j.jmatprotec.2012.11.022. 


\title{
Določanje optimalnih krajevno odvisnih sil pridrževanja pri globokem vleku $z$ uporabo metode odzivne površine
}

\author{
Volk, M. ${ }^{a,}{ }^{*}$, Nardin, B. ${ }^{a}$, Dolsak, B. ${ }^{b}$ \\ ${ }^{a}$ Gorenje Orodjarna, d.o.o., Velenje, Slovenia \\ ${ }^{b}$ University of Maribor, Faculty of Mechanical Engineering, Maribor, Slovenia
}

\section{POVZETEK}

Preoblikovanje pločevine je danes visoko avtomatiziran velikoserijski izdelovalni postopek za izdelavo različnih pločevinastih izdelkov. Maksimiranje kakovosti izdelka in posledično minimiziranje odpada ter proizvodnih stroškov so glavni cilji podjetij, ki se ukvarjajo s preoblikovanjem pločevine. Po drugi strani pa izdelki iz pločevine postajajo vse bolj zapleteni, predvsem zaradi čedalje zahtevnejših oblik in vse večje uporabe visokotrdnostnih pločevin, ki imajo manjšo zmožnost preoblikovanja. Zato se vedno pogosteje pojavlja potreba po optimizaciji postopka preoblikovanja pločevine. Ta raziskava prikazuje sistem za optimizacijo postopka preoblikovanja pločevine $\mathrm{z}$ uporabo metode končnih elementov (angl. Finite Element Method - FEM) v kombinaciji z metodo odzivne površine (angl. Response Surface Method RSM). Predlagan optimizacijski sistem smo preizkusili na praktičnem zgledu iz industrije gospodinjskih aparatov. V delu je opisano, kako določiti optimalno krajevno odvisno silo pridrževanja pri globokem vleku, da bi dobili najboljšo kakovost vlečenca. Optimizacijski sistem sestavljajo trije glavni koraki: modeliranje, presejanje (angl. Screening) ter optimizacija. Rezultati so pokazali, da je mogoče $\mathrm{z}$ boljšo konfiguracijo sil pridrževanja dobiti boljše rezultate. Pri optimizaciji so bili upoštevani tako preoblikovalni kriteriji kot tudi kriteriji elastičnega izravnavanja. Število potrebnih numeričnih simulacij se je $\mathrm{z}$ uporabo metode odzivne površine in načrtovanjem eksperimentov še zmanjšalo in je tako dosti manjše kot pri drugih običajnih optimizacijskih metodah. Zaradi tega lahko dobimo razmeroma natančen rezultat v kratkem času, kar je ena od glavnih prednosti te metode.

\section{PODATKI O ČLANKU}

Ključne besede:

Preoblikovanje pločevine

Optimizacija

Metoda končnih elementov

Metoda odzivne površine

*Kontaktna oseba:

mihael.volk@gorenje-orodjarna.si (Volk, M.)

Zgodovina članka:

Prejet 6. decembra 2013

Popravljen 30. maja 2014

Sprejet 3. junija 2014 